\title{
Clinical and Radiological Outcomes of Posterior Vertebral Column Resection for Severe Spinal Deformities
}

\author{
Byoung Hun Lee, M.D., ${ }^{1}$ Seung-Jae Hyun, M.D., Ph.D., Ki-Jeong Kim, M.D., Ph.D.,' Tae-Ahn Jahng, M.D., Ph.D., \\ Yongjung J. Kim, M.D., ${ }^{2}$ Hyun-Jib Kim, M.D., Ph.D.' \\ Department of Neurosurgery, Spine Center, Seoul National University Bundang Hospital, Seoul National University College of Medicine, \\ Seongnam, Korea \\ Department of Orthopaedic Surgery, ${ }^{2}$ Spine Service, Columbia University College of Physicians and Surgeons, New York, NY, USA
}

Objective : The aim of this study was to investigate clinical and radiological outcomes of patients who underwent posterior vertebral column resection (PVCR) by a single neurosurgeon in a single institution.

Methods : Thirty-four consecutive patients with severe spinal deformities who underwent PVCR between 2010 and 2016 were enrolled. The radiographic measurements included a kyphotic angle of PVCR levels (VCR angle), sagittal vertical axis (SVA), thoracic kyphosis, lumbar lordosis (LL), and spinopelvic parameters. The data of surgical time, estimated blood loss, duration of hospital stay, complications, intraoperative neurophysiologic monitoring, and the Scoliosis Research Society (SRS)-22 questionnaire were collected using a retrospective review of medical records.

Results : The VCR angle, LL, and SVA values were significantly corrected after surgery. The VCR and LL angle were changed from the average of $38.4 \pm 32.1^{\circ}$ and $-22.1 \pm 39.1^{\circ}$ to $-1.7 \pm 29.4^{\circ}(p<0.001)$ and $-46.3 \pm 23.8^{\circ}(p=0.001)$, respectively. The SVA was significantly reduced from $103.6 \pm 88.5 \mathrm{~mm}$ to $22.0 \pm 46.3 \mathrm{~mm}$ ( $p=0.001$ ). The clinical results using SRS-22 survey improved from $2.6 \pm 0.9$ to $3.4 \pm 0.8$ $(p=0.033)$. There were no death and permanent neurological deficits after PVCR. However, complications occurred in $19(55.9 \%)$ patients. Those patients experienced a total of 31 complications during- and after surgery. Sixteen reoperations were performed in twelve (35.3\%) patients. The incidence of transient neurological deterioration was 5.9\% (two out of 34 patients).

Conclusion : Severe spinal deformities can be effectively corrected by PVCR. However, the PVCR technique should be utilized limitedly because surgery-related serious complications are relatively common.

Key Words : Posterior · Vertebral column · Resection · Deformity · Complication.

\section{INTRODUCTION}

The vertebral column resection (VCR) is the most powerful surgical technique that removal of the entire vertebral body and the adjacent intervertebral discs to correct severe spinal deformities. It can be applied to fixed severe spinal deformi-

\footnotetext{
- Received : June 4, 2017 •Revised : July 31, 2017 •Accepted : August 30, 2017

- Address for reprints : Seung-Jae Hyun, M.D., Ph.D.

Department of Neurosurgery, Spine Center, Seoul National University Bundang Hospital, Seoul National University College of Medicine, 82 Gumi-ro 173beon-gil, Bundang-gu, Seongnam 13620, Korea

Tel : +82-31-787-7164, Fax : +82-31-787-4097, E-mail : hyunsj@snu.ac.kr
}

This is an Open Access article distributed under the terms of the Creative Commons Attribution Non-Commercial License (http://creativecommons.org/licenses/by-nc/4.0) which permits unrestricted non-commercial use, distribution, and reproduction in any medium, provided the original work is properly cited. 
ties such as congenital kyphoscoliosis, post-traumatic deformity, post-fusion flatback deformity and post-tuberculosis kyphosis $^{10)}$. The conventional VCR technique was performed by anterior- and posterior combined approaches ${ }^{2,3)}$. Suk et al. ${ }^{17)}$ have introduced posterior VCR (PVCR) procedure. Thereafter several researchers reported the outcomes following PVCR sequentially $^{12,17,18,20)}$. The PVCR has purposed at the decrease of complications and technical difficulties of the traditional anterior-posterior VCR ${ }^{17)}$. However, the PVCR is still a surgically demanding procedure because of the high risk of complications and operative hardship such as manipulation around the exposed spinal cord, resection of the posterior vertebral body, and the dissection problem with the opportunity of dural tears or neural injury ${ }^{5)}$. Nevertheless, the degree of correction is so powerful that it can be the most integral technique for severe spinal deformity ${ }^{5)}$. The aim of this study was to investigate clinical and radiological outcomes of patients who underwent PVCR by a single neurosurgeon in a single institution.

\section{MATERIALS AND METHODS}

\section{Demographics of patients}

This study protocol was approved by the institutional review board of our institution. A retrospective review of the patients was carried out in a single institution. Thirty-four consecutive patients with severe spinal deformities who underwent PVCR between December 2010 and June 2016 were enrolled in this study. All patients complained severe pain or neurological symptoms and progressing deformities that did not respond to conservative treatment. There were 13 males and 21 females with a mean age of $58.9 \pm 22.9$ years (range, 6-82). The patients were followed up for an average of $25.6 \pm 13.9$ months (range, 10-76) after surgery. The surgical indication for PVCR in this study were as follows : severe rigid or fixed deformity which should obtain a correction angle of 45 degrees or greater, 3 -dimensional multiplanar deformities, sharp angular deformities, and deformities that can't be corrected by other osteotomies (Smith-Petersen osteotomy and pedicle subtraction osteotomy). The diagnoses were a post-fusion flat back deformity, post-traumatic kyphosis, post-tuberculosis kyphosis, and congenital kyphoscoliosis (Fig. 1). Exclusion criteria included spinal infection, tumor, and a combined anterior-posterior VCR surgery. Seventeen patients had previously undergone spinal arthrodesis. The PVCR levels were 45 in total. The mean fused vertebrae were 9.6 \pm 3.8 (range, 5-17) (Table 1).

\section{Surgical procedure}

The surgical procedure was performed under general anesthesia and prone position on Jackson frame. All patients were used intraoperative neurophysiologic monitoring (IOM) (Xltek protektor 32 IOM system, Natus 142 Medical Inc., Oakvile, Canada) during surgery. After standard posterior subperiosteal dissection, pedicle screws (CD-Horizon Legacy screw system, Medtronic, Memphis, TN, USA) were placed using a freehand technique. Then, a short rod was temporarily installed to the pedicle screw above and below more two levels to provide stability during the PVCR. The medial ribs including rib head were removed from the thoracic spine, and the transverse process was resected from the lumbar spine to widen the field of view at the vertebrae where the PVCR was performed. After the proximal and distal junctional discs were removed, the osteotomy was started from the pedicle to the vertebral body. The exiting nerve root was tied and transected at the thoracic levels, whereas it was gently retracted at the lumbar levels. Because of the posterior wall of the vertebral body was maintained until the osteotomy is complete, the posterior wall was resected using a reverse cutting curette or Woodson dissector. After proper removal of the vertebral body, another rod was placed on the other side of the previously secured site. The deformity correction was gradually performed by compression and in situ contouring of the resected vertebral column using a pre-contoured permanent rods. In the maneuver, the surgeon must carefully confirm whether central canal and exiting nerve root were compressed or not and pay attention the IOM change during correction of the deformity. The defect gap was stuffed with a titanium mesh cage or a bone strut to achieve bony fusion. The final contour was secured with segmental rod fixation, and the multiple rods (3-4 rods) construct was usually utilized ${ }^{8)}$. After the final fixation procedure, posterior and posterolateral fusion was meticulously performed with a bunch of auto- and allografts.

\section{Radiological evaluation}

The radiologic measurements included a kyphotic angle of PVCR levels, sagittal balance, thoracic kyphosis (TK), and lumbar lordosis (LL). The methods were performed with the Cobb angle on preoperative and postoperative radiographs. The 
kyphosis angle of PVCR levels was defined between the superior endplate of the first vertebra above the PVCR and the inferior endplate of the first vertebra below the PVCR. The sagittal balance was measured by the horizontal distance from the $\mathrm{C} 7$ plumb line to the posterior-superior corner of S1 endplate on standing whole spine lateral radiographs. The angle between the superior endplate of T5 and the inferior endplate of T12 was determined TK. LL has used the angle from the superior endplate of L1 to the superior endplate of S1.

\section{Clinical evaluation}

The data of surgical time, estimated blood loss (EBL), duration of hospital stay, complications, and IOM data were collected by the retrospective review of patient charts. The Scoliosis Research Society (SRS)-22 questionnaire was used to evaluate clinical outcome. SRS-22 questionnaire was surveyed regularly (preoperative, postoperative six week, one year, two year, and so forth).
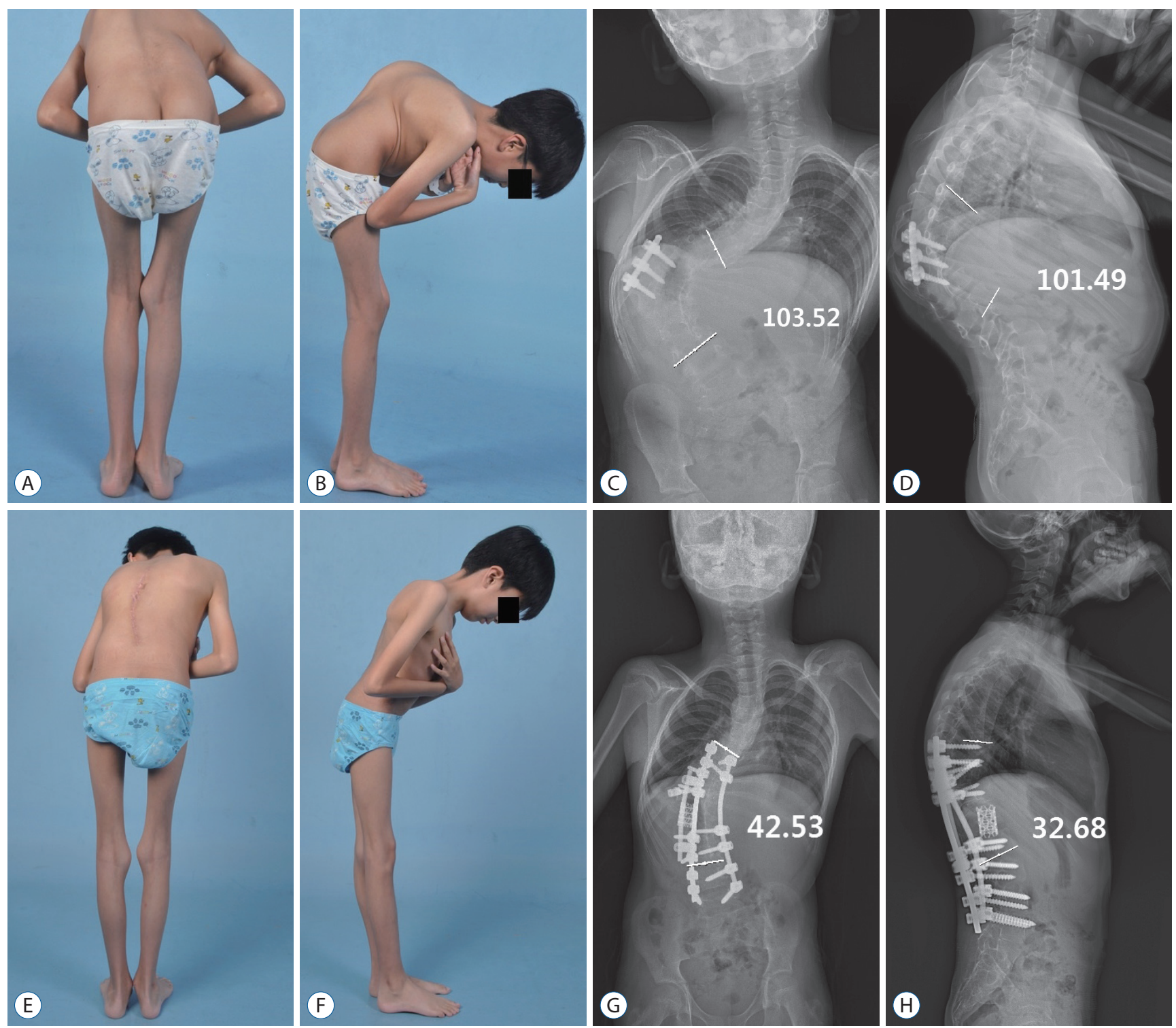

Fig. 1. An 11-year-old boy who suffered from early onset scoliosis. The left side rib hump was noted at preoperative clinical features (A and B). The preoperative postero-anterior (C) and lateral (D) radiographs showed severe kyphoscoliosis of more than 100 degrees and prior arthrodesis at T11-L1. The patient has received an apical PVCR at T11-L1, and posterior spinal fusion from T8 to L5 with multiple rods construct. The correction of coronal and sagittal alignment maintained well on the clinical pictures ( $E$ and $F$ ) and radiographs ( $G$ and $H)$ six months after surgery. PVCR : posterior vertebral column resection. 


\section{Statistical analysis}

Statistical data analysis was carried out using SPSS Statistics version 20.0 (IBM Corp., Armonk, NY, USA). Paired t-test was used to estimate the effectiveness of PVCR by comparison of the Cobb angle before and after surgery. The Pearson correlation test was selected for analysis of differences between clinical factors and complications. The $p$-value $<0.05$ considered as the statistical significance.

\section{RESULTS}

The correction of VCR angle improved from the average of $38.4 \pm 32.1^{\circ}$ to $-1.7 \pm 29.4^{\circ}(p<0.001)$. Lumbar lordosis changed from

Table 1. Demographics of all patients

\begin{tabular}{lc}
\hline Type & Value \\
\hline Sex (F/M) & $21 / 13$ \\
Age & $58.9 \pm 22.9$ (range, 6-82) \\
Follow-up duration (months) & $25.6 \pm 13.9$ (range, 10-76) \\
Diagnosis & 16 \\
Post-fusion flat back deformity & 7 \\
Post-traumatic kyphosis & 6 \\
Post-tuberculous kyphosis & 5 \\
Congenital kyphoscoliosis & 17 \\
Prior operation history (spine) & \\
Resection levels & 14 \\
Thoracic (T3-12) & 31 \\
Lumbar (L1-5) & 45 \\
Total & $9.6 \pm 3.8$ (range, 5-17) \\
Fused levels &
\end{tabular}

F: female, $M$ : male the mean $-22.1 \pm 39.1^{\circ}$ to $-46.3 \pm 23.8^{\circ}$ at the follow-up $(p=0.001)$. The average angle change in TK and pelvic incidence (PI) revealed from the median $27.9 \pm 28.5^{\circ}$ and $50.8 \pm 11.5^{\circ}$ to $32.1 \pm 16.2^{\circ}$ $(p=0.611)$ and $50.5 \pm 10.9^{\circ}(p=0.291)$, respectively. The sagittal vertical axis (SVA) were significantly reduced from 103.6 \pm 88.5 $\mathrm{mm}$ to $22.0 \pm 46.3 \mathrm{~mm}(p<0.001)$ (Table 2).

The clinical results using SRS-22 score significantly improved from 2.6 \pm 0.9 to $3.4 \pm 0.8$ after surgery ( $p=0.033$ ) (Table 3$)$. The average surgical time was $386.5 \pm 114.8$ minutes, and mean hospital stay was $17.9 \pm 10.9$ days after PVCR. The median EBL was $1134.8 \pm 1149.9 \mathrm{~mL}$ and transfusion was mean $975.8 \pm 960.5 \mathrm{~mL}$. Duration of hospital stay was significantly longer in the patients having perioperative complications $(p=0.009)$, whereas other surgical parameters did not have a significant relationship with the complications.

The complications were verified in 19 (55.9\%) patients. A total of 31 complications occurred during and after surgery (Table 4). Sixteen reoperations were performed after PVCR in twelve (35.3\%) patients. IOM changes during surgery observed in six patients (17.6\%). Four patients $(66.7 \%)$ out of those were fully recovered intra-operatively. However, deteriorated IOM changes of the remaining two patients $(33.3 \%)$ were not restored. The patients experienced postoperative transient paresis and recovered later. Intraoperative complications occurred in four patients. There were 17 cases of postoperative compli-

Table 3. Clinical outcomes of PVCR

\begin{tabular}{lccc}
\hline Clinical outcomes & Pre-operation & Post operation & $p$-value \\
\hline SRS-22 score & $2.6 \pm 0.9$ & $3.4 \pm 0.8$ & $0.033^{*}$ \\
\hline
\end{tabular}

${ }^{*} p$-value $<0.05$. PVCR : posterior vertebral column resection, SRS : Scoliosis Research Society

Table 2. Radiologic outcomes of PVCR

\begin{tabular}{lccc}
\hline Global sagittal balance parameter & Pre-operation & Follow-up & $p$-value \\
\hline Pelvic incidence $\left(^{\circ}\right)$ & $50.8 \pm 11.5$ & $50.5 \pm 10.9$ & 0.291 \\
\hline Sagittal vertical axis $(\mathrm{mm})$ & $103.6 \pm 88.5$ & $22.0 \pm 46.3$ & $<0.001^{*}$ \\
\hline Thoracic kyphosis $\left(^{\circ}\right)$ & $27.9 \pm 28.5$ & $32.1 \pm 16.2$ & 0.611 \\
Lumbar lordosis $\left(^{\circ}\right)$ & $-22.1 \pm 39.1$ & $-46.3 \pm 23.8$ & $0.001^{*}$ \\
\hline Pelvic tilt $\left(^{\circ}\right)$ & $27.2 \pm 13.5$ & $17.8 \pm 11.2$ & $0.000^{*}$ \\
\hline Sacral slope $\left(^{\circ}\right)$ & $23.6 \pm 16.0$ & $32.7 \pm 15.1$ & $0.003^{*}$ \\
PI-LL $\left(^{\circ}\right)$ & $25.6 \pm 18.4$ & $11.2 \pm 10.4$ & $0.000^{*}$ \\
$\operatorname{VCR}$ angle $\left(^{\circ}\right)$ & $38.4 \pm 32.1$ & $-1.7 \pm 29.4$ & $<0.001^{*}$ \\
\hline
\end{tabular}

${ }^{*} p$-value $<0.05$. PVCR : posterior vertebral column resection, PI : pelvic incidence, LL : lumbar lordosis, VCR : vertebral column resection 
cations within two weeks. The late-onset complications developed in the remaining 10 patients more than two weeks after surgery.

\section{Intraoperative complications}

There were two cases of excessive intraoperative bleeding. One case of bleeding $(>3000 \mathrm{~mL}$ ) has an unstable vital sign during surgery. In the other case, there was a massive bleeding of $5000 \mathrm{~mL}$ or more. The patients with excessive bleeding recovered uneventfully after receiving intensive postoperative

Table 4. Complications and reoperations of PVCR

\begin{tabular}{|c|c|}
\hline Type & $\begin{array}{c}\text { Complications } \\
\text { (19 patients) }\end{array}$ \\
\hline Dura tear & 2 \\
\hline Delayed CSF leakage & 2 \\
\hline Massive bleeding (>5000 mL) & 1 \\
\hline Excessive bleeding with unstable vital sign (>3000 mL) & 1 \\
\hline Root compression by bone fragment & 1 \\
\hline Neurologic deficiency & 2 \\
\hline Cervical myelopathy & 1 \\
\hline Surgical site infection-superficial & 2 \\
\hline Wound dehiscence & 3 \\
\hline Urinary tract infection & 1 \\
\hline Fecal incontinence & 1 \\
\hline Pneumonia & 2 \\
\hline Delirium & 2 \\
\hline \multicolumn{2}{|l|}{ Adjacent segment fracture } \\
\hline UIV+1 & 2 \\
\hline UIV+3 & 1 \\
\hline LIV & 1 \\
\hline LIV+1 & 1 \\
\hline Screw loosening & 1 \\
\hline Screw malposition & 2 \\
\hline Remnant curve progression & 1 \\
\hline Proximal junctional kyphosis & 1 \\
\hline Total complications number & 31 \\
\hline Reoperation & 16 \\
\hline IOM change in operation & 6 \\
\hline Recovery & 4 \\
\hline Non-recovery & 2 \\
\hline
\end{tabular}

PVCR : posterior vertebral column resection, CSF : cerebrospinal fluid, UIV : upper instrumented vertebra, LIV : lower instrumented vertebra, $\mathrm{IOM}$ : intraoperative neurophysiologic monitoring care with blood transfusion. The dura tears occurred in two patients. The patients underwent primary closure without additional leakage or revision surgery.

\section{Postoperative complications ( $<2$ weeks)}

There was two delayed cerebrospinal fluid leakage without revision surgery. In one patient, nerve root irritation symptoms were severe after the PVCR. The nerve root was compressed by bone fragments, and reoperation was carried out. It had not detected by IOM during the initial surgery. The patients recovered without neurological deficit. Two patients had postoperative transient neurological deficits. They complained of substantial motor weakness at lower extremities after surgery. The patients underwent revision surgery, one had compression of the thecal sac by remnant bone fragment, and the other had massive bleeding during surgery. However, they fully recovered through conservative treatment after 2nd surgery. There was a patient having myelopathic symptoms of the cervical spine after PVCR surgery. In the patient, a stenotic lesion at the cervical 34 level was overlooked. The myelopathy improved after additional cervical surgery. There were two cases of superficial surgical site infection and three of wound dehiscence. The wound dehiscence patients have received reoperation, and superficial surgical site infection patients underwent conservative treatment. Other complications such as pneumonia, delirium, urinary tract infection, and fecal incontinence were also developed, however those were treated by conservative managements.

\section{Late onset complications ( $\geq 2$ weeks)}

Five patients suffered from a compression fracture of the vertebral body during the follow-up (from 1 month to 6 months after surgery). Three and two out of those occurred at upper instrumented vertebra (UIV) and lower instrumented vertebra (LIV), respectively. Vertebroplasty was carried out to two patients having UIV fracture. Screw related complications occurred in three patients. Screw malposition (two patients) and screw loosening (one patient) were symptomatic requiring reoperation. Kyphosis progression occurred in a patient (two years later after surgery). The patient experienced proximal junctional kyphosis requiring additional surgery for fusion extension. One patient suffered from thoracic decompensation with remant curve progression. The patient had connective tissue disease of Loeys-Dietz syndrome. 


\section{DISCUSSION}

Severe spinal deformities may cause a hazard to adjacent spinal cord and nerves, impaired general health such as respiratory function, and profound impact on cosmetic appearance ${ }^{14,21}$. The deformity corrective surgery may be necessary in selective cases. However, it is a challenging procedure because of its high complication rate and surgical difficulty ${ }^{6,9)}$. In our series, the PI-LL was mean $25.4 \pm 18.6^{\circ}$ and SVA was mean $103.6 \pm 88.5 \mathrm{~mm}$ before surgery. These results were considered as a condition of severe deformity according to SRS-Schwab classification ${ }^{19)}$. Furthermore, PVCR was limitedly indicated for severe fixed deformity, 3-dimensional multiplanar deformities, sharp angular deformities in this study. Thesedays, PVCR was widely utilized for fixed severe spinal deformities instead of the conventional circumferential VCR ${ }^{16,17)}$. PVCR had several advantages comparing to traditional anterior VCR such as effective correction of the deformity as well as a reduction of operative time, blood loss, complications ${ }^{17}$. In the present study, the VCR angle, LL, and SVA values were significantly corrected after surgery. Clinical outcomes in terms of SRS-22 scores after PVCR were quite satisfactory. However, PVCR has a high complication rate in several previous studies ${ }^{7,11,16,17,22)}$. In the current study, 19 (55.9\%) of 34 patients experienced a total of 31 complications. The prevalence of transient neurological complications was 5.9\% (two of 34 patients). Previous researchers reported that incidence of the neurological problem was from $1.2 \%$ to $17.1 \% \%^{7,11,16,17,22)}$. The mechanism of neurological deterioration as a critical complication after VCR was ischemic injury and mechanical damage to the spinal cord ${ }^{15)}$. In this study, one patient experienced lower extremities paraparesis after L5 PVCR surgery. It was confirmed that the thecal sac was mechanically compressed by remnant bone fragment of the L5 vertebral body in the field of reoperation. For this reason, surgeons need to be very careful when performing the compression of a PVCR site. The other patient had massive bleeding during surgery with an unstable vital sign. Estimated blood loss was about $3000 \mathrm{~mL}$. Mean arterial pressure (MAP) was dropped to $60 \mathrm{mmHg}$ due to bleeding during surgery and motor evoked potential signal lost. Smith et al. ${ }^{15)}$ described that blood hypoperfusion of the spinal cord by intraoperative hemorrhage are an immediate cause of neurological complications. Previous investigators suggested that more than 2000 $\mathrm{mL}$ is a risk factor for the problems $\mathrm{s}^{1,11}$. Lenke and colleagues ad- vocated that MAP should be sustained from 85 to $90 \mathrm{mmHg}$ at the close the osteotomy site ${ }^{13)}$. In the current series, the target MAP level was maintained above $85 \mathrm{mmHg}$. Moreover, we routinely used cell saver and tranexamic acid (TXA) during surgery to prevent excessive bleeding and allogenic transfusion. Choi et al. ${ }^{4}$ reported that TXA could decrease intraoperative bleeding by $37 \%$ of mean EBL. In this series, IOM was applied to all patients, and IOM change was carefully monitored during surgery. In this study, six patients of IOM changes were detected, and when this change confirmed, we tried to stop the surgery and to correct the cause. Despite careful attention during surgery, there is various surgery related complications in our series. Hypoperfusion to the spinal cord due to bleeding during surgery and unexpected nerve injury owing to spinal cord distraction or compression by bony fragments are the main causes of neurological complications following PVCR. Meticulous attention should be paid to patients who have sharp angular kyphosis such as tuberculosis kyphosis and unusual anatomical structure owing to multiple prior surgeries. The compression fracture of vertebral body was developed at UIV and LIV level. Screw loosening and proximal junctional kyphosis also occurred for follow-up period. Because the surgical wound is wide, infection and dehiscence should not be underestimated. We had five wound-related problems. Since the postoperative systemic disease can frequently occur such as pneumonia and urinary tract infection, surgeons need to take a meticulous care of the patient after surgery.

Several limitations deserve to be mentioned in this study. It has a retrospective research design with a small sample size. Another limitation is that there are not a few patients (17 patients) who had undergone prior spine surgery in the study population. It is thought to affect the occurrence of complications.

\section{CONCLUSION}

Severe spinal deformities can be effectively corrected by PVCR. Radiographic and clinical outcomes after PVCR were quite satisfactory. However, the PVCR technique should be utilized limitedly because surgery-related serious complications are relatively common. 


\section{CONFLICTS OF INTEREST}

The authors have no financial conflicts of interest.

\section{INFORMED CONSENT}

This type of study does not require informed consent.

\section{References}

1. Auerbach JD, Lenke LG, Bridwell KH, Sehn JK, Milby AH, Bumpass $D$, et al. : Major complications and comparison between 3-column osteotomy techniques in 105 consecutive spinal deformity procedures. Spine (Phila Pa 1976) 37 : 1198-1210, 2012

2. Boachie-Adjei 0, Bradford DS : Vertebral column resection and arthrodesis for complex spinal deformities. J Spinal Disord 4 : 193-202, 1991

3. Bradford DS, Tribus CB : Vertebral column resection for the treatment of rigid coronal decompensation. Spine (Phila Pa 1976) 22 : 1590-1599, 1997

4. Choi HY, Hyun SJ, Kim KJ, Jahng TA, Kim HJ : Effectiveness and safety of tranexamic acid in spinal deformity surgery. J Korean Neurosurg Soc $60: 75-81,2017$

5. Demirkiran G, Dede O, Karadeniz E, Olgun D, Ayvaz M, Yazici M : Anterior and posterior vertebral column resection versus posterior-only technique: a comparison of clinical outcomes and complications in congenital kyphoscoliosis. Clin Spine Surg 30 : 285-290, 2016

6. Enercan M, Ozturk C, Kahraman S, Sarier M, Hamzaoglu A, Alanay A : Osteotomies/spinal column resections in adult deformity. Eur Spine J 22 Suppl 2 : S254-S264, 2013

7. Hamzaoglu A, Alanay A, Ozturk C, Sarier M, Karadereler S, Ganiyusufoglu K : Posterior vertebral column resection in severe spinal deformities: a total of 102 cases. Spine (Phila Pa 1976) 36 : E340-E344, 2011

8. Hyun SJ, Lenke LG, Kim YC, Koester LA, Blanke KM : Comparison of standard 2-rod constructs to multiple-rod constructs for fixation across 3-column spinal osteotomies. Spine (Phila Pa 1976) 39 : 1899-1904, 2014

9. Hyun SJ, Rhim SC : Clinical outcomes and complications after pedicle subtraction osteotomy for fixed sagittal imbalance patients: a long-term follow-up data. J Korean Neurosurg Soc 47 : 95-101, 2010

10. Kawahara N, Tomita K, Baba H, Kobayashi T, Fujita T, Murakami H : Clos- ing-opening wedge osteotomy to correct angular kyphotic deformity by a single posterior approach. Spine (Phila Pa 1976) 26 : 391-402, 2001

11. Lenke LG, Newton PO, Sucato DJ, Shufflebarger HL, Emans JB, Sponseller PD, et al. : Complications after 147 consecutive vertebral column resections for severe pediatric spinal deformity: a multicenter analysis. Spine (Phila Pa 1976) 38 : 119-132, 2013

12. Lenke LG, O'Leary PT, Bridwell KH, Sides BA, Koester LA, Blanke KM : Posterior vertebral column resection for severe pediatric deformity: minimum two-year follow-up of thirty-five consecutive patients. Spine (Phila Pa 1976) 34 : 2213-2221, 2009

13. Lenke $L G$, Sides BA, Koester LA, Hensley M, Blanke KM : Vertebral column resection for the treatment of severe spinal deformity. Clin Orthop Relat Res 468 : 687-699, 2010

14. Liu C, Lin L, Wang W, Lv G, Deng Y : Long-term outcomes of vertebral column resection for kyphosis in patients with cured spinal tuberculosis: average 8-year follow-up. J Neurosurg Spine 24 : 777-785, 2016

15. Smith JS, Wang VY, Ames CP : Vertebral column resection for rigid spinal deformity. Neurosurgery 63(3 Suppl) : A177-A182, 2008

16. Suk SI, Chung ER, Kim JH, Kim SS, Lee JS, Choi WK : Posterior vertebral column resection for severe rigid scoliosis. Spine (Phila Pa 1976) 30 : 1682-1687, 2005

17. Suk SI, Kim JH, Kim WJ, Lee SM, Chung ER, Nah KH : Posterior vertebral column resection for severe spinal deformities. Spine (Phila Pa 1976) 27 : 2374-2382, 2002

18. Tao Y, Wu J, Ma H, Zhang L, Shao S, Si Z, et al. : Posterior vertebral column resection for severe and rigid spinal deformity associated with neurological deficit after implant removal following posterior instrumented fusion: a case report and literature review. Spine (Phila Pa 1976) 40 : E794-E798, 2015

19. Terran J, Schwab F, Shaffrey Cl, Smith JS, Devos P, Ames CP, et al. : The SRS-Schwab adult spinal deformity classification: assessment and clinical correlations based on a prospective operative and nonoperative cohort. Neurosurgery $73:$ 559-568, 2013

20. Xie JM, Zhang Y, Wang YS, Bi N, Zhao Z, Li T, et al. : The risk factors of neurologic deficits of one-stage posterior vertebral column resection for patients with severe and rigid spinal deformities. Eur Spine J 23 : 149156, 2014

21. Yang C, Zheng Z, Liu H, Wang J, Kim YJ, Cho S : Posterior vertebral column resection in spinal deformity: a systematic review. Eur Spine J 25 : 2368-2375, 2016

22. Zhang BB, Zhang T, Tao HR, Wu TL, Duan CG, Yang WZ, et al. : Neurological complications of thoracic posterior vertebral column resection for severe congenital spinal deformities. Eur Spine J 26 : 1871-1877, 2017 